\title{
From the 16th to the 21st Century: how we approach mental health problems and where do we go next?
}

\author{
P. Dazzan ${ }^{1,2 *}$ and C. Barbui ${ }^{3}$ \\ ${ }^{1}$ Department of Psychosis Studies, King's College London, Institute of Psychiatry, Psychology and Neuroscience, London, UK \\ ${ }^{2}$ National Institute for Health Research (NIHR) Mental Health Biomedical Research Centre at South London and Maudsley NHS Foundation Trust \\ and King's College London, London, UK \\ ${ }^{3}$ Department of Public Health and Community Medicine, Section of Psychiatry, University of Verona, Verona, Italy
}

The first Editorial of this issue, by Ventriglio \& Bhugra (2015), discusses the influence of the 16th century philosopher Descartes and his concept of mind-body dualism on how we have conceptualized Western psychiatry. The authors state that this dualism has implied a lack of connection between mind and body, as if these worked in totally separate spheres, and propose that, along the centuries, this position has continued to plague modern medicine as well as psychiatry. They go further in stating that Descartes view of body and mind as separate has taken the form of a dogma, since people have continued to believe in it despite evidence showing the contrary.

The body-mind dualism has become increasingly challenged by scientific evidence, as we now understand more about the biological correlates of mental health disorders, with models that link mind and body. And yet, the mind-body split seems to still influence psychiatry training and service organisation. The authors quote the example of other health models, like the Ayurvedic one, which have conceptualised the individual in its entirety and as affected by a number of factors, and which propose a view of disorders of mind and body as interdependent. In psychiatry, they argue, a similar view would have left the door open to different management and therapeutic approaches. Instead, the body-mind dualism accepted in western health care may have caused a number of difficulties. These include the separation of psychiatric from medical care; the perception that nonpsychiatrists do not need knowledge of mental health disorders; and similarly, that psychiatrists tend to ignore physical health problems in their patients. The authors suggest that, worryingly, this view has contributed to the current inequality in health service provision and in research funding for psychiatry, which ignores

* Address for correspondence: Dr Paola Dazzan, Department of Psychosis Studies, PO40, Institute of Psychiatry, Psychology and Neuroscience, King's College London, De Crespigny Park, London SE5 8AF, UK.

(Email: paola.dazzan@kcl.ac.uk) that the burden of mental health disorder is larger than the one caused by heart disease or cancer. The Editorial finishes by arguing that it is now time to abandon this faux mind-body dualism in our training of physicians, and move towards a better integration between physical and mental illnesses in service provision.

Samele and Urquia, in the second Editorial, move the discussion on service provision to the next level, provocatively asking what is the future of psychiatric inpatient care, in view of the continuous decline in the number of hospital beds that has taken place across European countries (EU) (Samele \& Urquia, 2015). These are the countries that, one would assume, share a similar vision of mental health disorders. The number of psychiatric hospital beds across $28 \mathrm{EU}$ countries has in fact declined from 97.3 per 100000 population in 2000, to 68.5 in only 2001 (Eurostat, 2015). In addition to the decline, the enormous variability in hospital beds provision throughout these countries is also extremely interesting. This varies from a country like Italy having as little as 10 beds per 100000 population, to the UK having 53 beds per 100000 population, to countries like Belgium with 175 beds per 100000 population.

Large variability is also seen in number of hospital admissions. The Editorial reports figures provided by the World Health Organization (WHO) European Hospital Morbidity Database (European Hospital Morbidity Database, 2015) for 12 EU Member States. According to these, the number of inpatient admissions for patients with a diagnosis of schizophrenia ranges from 0.02 to 3.1 per 1000 population across these states. Interestingly, the UK seems to have one of the lowest admission rates, at 0.29 per 1000 population. However, when one looks at the length of these admissions, the UK also has one of the longest in-patient stays compared with the other countries, with an average of 131.7 days, 22 days longer than what it was just in 2012.

The reasons for this long duration of inpatient admissions remain unclear, as only few studies have looked at patterns and predictors of length of stay. 
Factors that have been quoted include cultural factors, the structure of mental health services and the pressure to discharge patients early (Sytema et al. 2002; Auffarth et al. 2008). The lack of community mental health services and lack of suitable accommodation have also been associated with longer length of stay (Zhang et al. 2011). It is intriguing, considering what highlighted by the Editorial of Ventriglio and Bhugra, that the presence of severe medical co-morbidities has been associated with longer stay in patients with schizophrenia, and prolonged by a median of 13 days. This highlights again the need for integrating physical and mental care.

In parallel with the increase in duration of hospitalisation, the last two decades have also witnessed an increase in the rates of compulsory admissions, possibly because, in order to be admitted, patients need to present with very severe symptoms. This increase has taken place even in countries where communitybased services are well developed, like the UK. In fact, they report how evidence suggests that the use of community specialist or crisis resolution services like those for early intervention in psychosis can reduce the number of hospital admissions and the duration of inpatient stay. The authors conclude highlighting the need for further research in what factors can affect length of stay as these could inform future decisions in policy and clinical practice.

These editorials provide an excellent critical and stimulating discussion on the difficulties we face in the integration between mental and physical health, and on the information we are missing to inform how we should organize and deliver mental and physical care in the future.

Some guidance is however available. As recently pointed out by a thematic paper generated by the Gulbenkian Mental Health Platform and the WHO (2014), integration is a concept that refers to how health care systems implement a response to mental disorders and other chronic disorders. Mental and physical disorders share common biological, genetic, psychological, social and environmental factors (Fig. 1) that should be addressed by appropriate health promotion strategies and disease prevention programmes. At this level, dichotomy between mind and body, or mental and physical, makes little sense, as these determinants may act alone or in different combinations, being simultaneously risk factors for mental and physical disorders. The WHO strongly supports integrated policies and plans to meet the mental and physical health and social needs of people at all stages of life, including infancy, childhood, adolescence, adulthood and old age.

Besides, appropriate services should be developed to provide integrated care for people who have already developed a disorder, with the aim of decreasing the consequences that, inevitably, reflect a combination of mental, physical, social and economic factors (Fig. 1). An integrated response, therefore, requires a multisectoral approach, and cannot be limited to the functioning of one mental health sector only. The WHO calls for redesigning health systems and services towards truly integrated care, with advantages not only in terms of meeting the overall health needs of those that require them, but also in terms of feasibility, efficiency and sustainability of the whole process of care in the long-term.

While access to physical care needs to be improved, it is essential we continue to provide access to good quality mental health care. The reduction in hospital beds of recent years makes one wonder whether in-patient care is made available when needed. In the UK, the provision of mental health beds has decreased by more than $60 \%$ between 1988 and 2008 (Keown et al. 2011). In addition, many in-patient wards now regularly have a 100-120\% occupancy rate, which is significantly higher than the $85 \%$ recommended by the Royal College of Psychiatrists (McNicoll, 2013). The reduction in beds has been matched by a parallel, greater than $60 \%$ increase in involuntary admissions during the same period, which does not seem to be matched by an increase in national mental health disorders and is possibly related to increase symptom severity at the time of presentation (Keown et al. 2011).

The fact that in many Western countries funding for mental health care is being cut significantly more than funding for physical care raises again the issue of where the mind-body divide leaves us: how has this concept influenced our perception of mental illness and the way we should care for this. Only 2 years ago, Thornicroft and Tansella [founding Editor of Epidemiology and Psychiatric Sciences who prematurely left us this year] summarised elegantly and poignantly the challenges faced by the provision of community-based psychiatric care (Thornicroft \& Tansella, 2014). In this context, they made a list of nine proposals to look into its future. The issues we have debated above feature prominently among others in their list: the need to recognise the shorter life expectancy of individuals with mental health problems and to evaluate methods that reduce this disparity; the need to reduce the stigma and discrimination perceived by people with mental health problems; and the need for mental health care to provide a 'careful balance' of hospital and community care. We would like to finish with their words, that 'In all countries, a very substantial challenge exists to make a long-term commitment to improve the acceptance among the general population of people with mental illness and to end, within our 


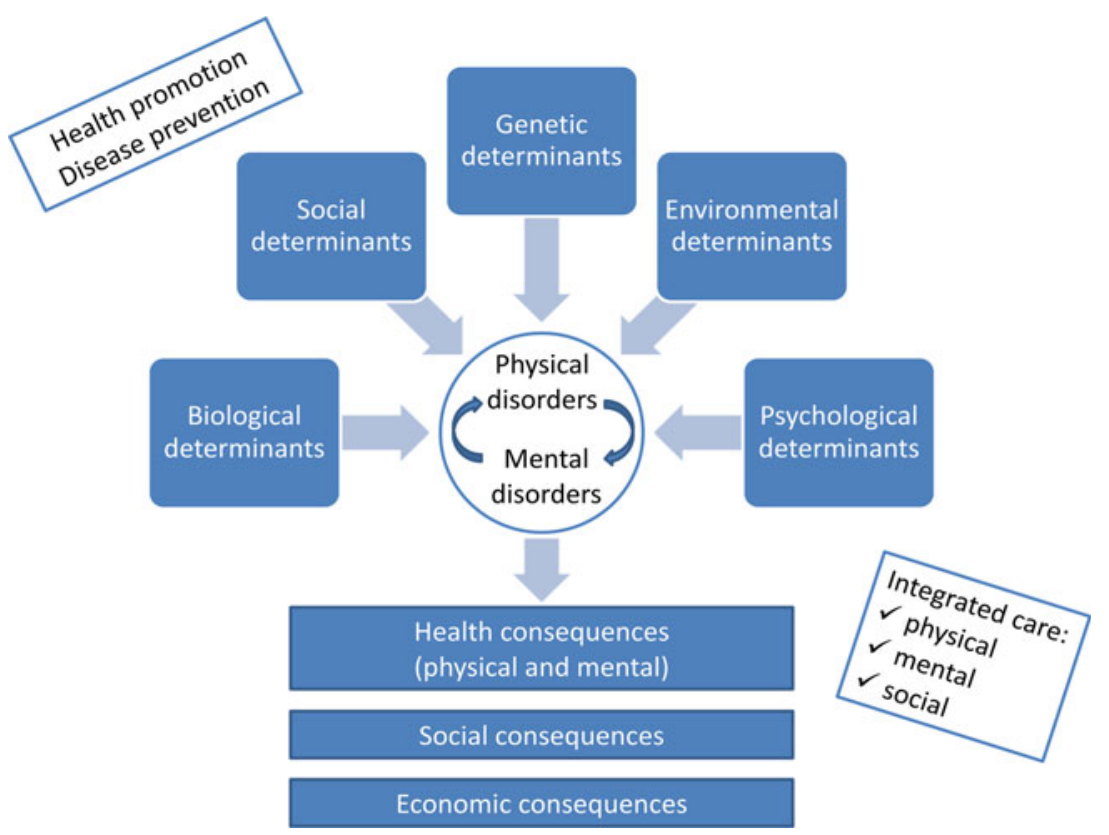

Fig. 1. Mental and physical disorders are highly interdependent in terms of risk factors and consequences, requiring integrated health promotion and prevention policies, together with appropriate health and social care plans.

lifetimes, the systematic social exclusion that has left people with mental illness outside the mainstream of our communities and societies.' Albeit challenging, we hope this will indeed be achieved within our lifetimes.

\section{Financial Support}

This research received no specific grant from any funding agency, commercial or not-for-profit sectors.

\section{Conflict of Interest}

None.

\section{References}

Auffarth I, Busse R, Dietrich D, Emrich H (2008). Length of psychiatric inpatient stay: comparison of mental health care outlining a case mix from a hospital in Germany and the United States of America. German Journal of Psychiatry 11, 40-44.

European Hospital Morbidity Database (2015). World Health Organization Regional Office for Europe. Retrieved March 2015 from http://data.euro.who.int/hmdb/.

Eurostat (2015). Psychiatric Care Beds in Hospitals. Retrieved March 2015 from http://ec.europa.eu/eurostat/tgm/table.do? tab $=$ table\&plugin $=1 \&$ language $=$ en\&pcode $=$ tps 00047 .
Keown P, Weich S, Bhui K, Scott J (2011). Association between provision of mental illness beds and rate of involuntary admissions in the NHS in England 1988-2008: ecological study. British Medical Journal 343, d3736.

McNicoll A (2013). Patients at risk as 'unsafe' mental health services reach crisis point. Community Care investigation prompts minister to pledge to end "institutional bias against mental health" in NHS Psychiatric inpatient care: where do we go from here? Community Care October 16, 2013.

Samele C, Urquía N (2015). Psychiatric inpatient care: where do we go from here? Epidemiology and Psychiatric Sciences. doi: 10.1017/S2045796015000591.

Sytema S, Burgess P, Tansella M (2002). Does community care decrease length of stay and risk of rehospitalisation in new patients with schizophrenia disorder. Schizophrenia Bulletin 28, 273-281.

Thornicroft G, Tansella M (2014). Community mental health care in the future: nine proposals. Journal of Nervous and Mental Disease 202, 507-512.

Ventriglio A, Bhugra D (2015). Descartes' dogma and damage to Western psychiatry. Epidemiology and Psychiatric Sciences. doi: 10.1017/S2045796015000608.

World Health Organization and Calouste Gulbenkian Foundation (2014). Integrating the Response to Mental Disorders and other Chronic Diseases in Health Care Systems. World Health Organization: Geneva.

Zhang J, Harvey C, Andrew C (2011). Factors associated with length of stay and the risk of readmission in an acute psychiatric inpatient facility: a retrospective study. Australian and New Zealand Journal of Psychiatry 45, 578-585. 